\title{
Bubbles in viscous liquids: time dependent behaviour and wake characteristics
}

\author{
Monica Gumulya ${ }^{1 *}$, Jyeshtharaj B. Joshi ${ }^{2,3}$, Ranjeet P. Utikar ${ }^{1}$, \\ Geoffrey M. Evans ${ }^{4}$, Vishnu Pareek ${ }^{1}$ \\ ${ }^{1}$ Department of Chemical Engineering, Curtin University, U1987, Perth 6845, WA, AUSTRALIA \\ ${ }^{2}$ Homi Bhabha National Institute, Anushaktinagar, Mumbai-400 094, India \\ ${ }^{3}$ Department of Chemical Engineering, Institute of Chemical Technology, Matunga, Mumbai- \\ 400019. \\ ${ }^{4}$ Discipline of Chemical Engineering, The University of Newcastle, NSW, 2308, Australia \\ *Corresponding author, E-mail address: m.gumulya@curtin.edu.au
}

\begin{abstract}
The dynamics of a bubble, initially stationary and spherical, rising in a viscous Newtonian liquid have been studied numerically using 3-D Volume-of-Fluid (VOF) method implemented in the Gerris flow solver. The study encompasses $8.7 \leq E O$ ( $\left.=\Delta \rho g D^{2} / \sigma\right) \leq 641$ and $R e \leq 151$. Additionally, results published in the literature encompassing bubbles with lower values of Eo numbers were also considered, such that the overall dependencies of bubble shape, wake characteristics, and drag coefficient over a large range of $E o$ and $R e$ values can be identified. While it was found that the deformation of the bubbles as predicted through the numerical study can generally replicate experimental observations presented, several limitations were identified, such as in the representation of skirt formation behind a skirted bubble and the formation of satellite bubbles behind a bubble rising at high Reynolds numbers. The dependency of the bubble aspect ratio on the Weber and Morton numbers was confirmed for cases of spherical and ellipsoidal bubbles; whilst for spherical cap and skirted bubbles the aspect ratio was found to depend largely on the Reynolds and Capillary numbers, respectively. Finally, the expansion and formation of closed/open laminar wakes behind the rising bubble were analysed and was found to correlate well with the bubble $R e$ and $E O$ numbers.
\end{abstract}

Keywords: Volume-of-fluid method; CFD, bubble shape, bubble rise velocity; drag force.

\section{Introduction}

The motion of bubbles in fluids is of great importance in various gas-liquid reactors and processes, as well as numerous natural phenomena. As a result, extensive studies have been conducted in the past (see reviews by Clift, Grace, and Weber (1978), Magnaudet \& Eames (2000), and Kulkarni \& Joshi (2005)), although various aspects still remain indeterminate, particularly in relation to the dynamic behaviour of the bubbles in liquids. The complexity of the rise of bubbles is brought about by its dependence on several factors: their buoyancy and the external and internal circulations of the two fluids, which dictate the inertial and viscous forces experienced by the bubbles, as well as the interfacial forces between the two phases, i.e. surface tension and the presence of 
impurities/surfactants in the system. Consequently, numerous bubble shapes can be obtained. Relatively small bubbles rising in viscous fluids generally attain a spherical shape as their hydrodynamics are largely dominated by the surface tension and the viscous forces of the liquid phase. In fluids of lower viscosities, inertial forces can become more dominant and the bubbles can no longer retain their spherical shape thus exhibiting more oblate ellipsoidal shapes. Further instabilities may occur if the fluid viscosity is reduced even further, resulting in a wobbling/zig-zag motion in the rise of the bubbles. The behaviours of spherical and ellipsoidal bubbles have been studied experimentally and numerically by Raymond \& Rosant (2000); whilst the onset of path instabilities has been studied experimentally by Zenit \& Magnaudet (2009) and numerically by Cano-Lozano et al. (2013). With larger bubbles the inertial forces caused by their heightened buoyancy tend to cause a loss in fore-and-aft symmetry, and the bubbles tend to adopt a spherical cap shapes. In some cases, the rim of the rear of the bubble may become drawn out due to the viscosity of the fluids, and the bubbles develop "skirts" and appear to have an elongated section in their wake.

The different shapes of bubbles rising in a stagnant fluid have been demonstrated experimentally in the work of Bhaga \& Weber (1981). Along with the shapes of the bubble wakes and the general features of the velocity streamlines, they also measured the drag experienced by the bubbles and their respective terminal velocities. Not surprisingly, the work of Bhaga \& Weber (1981) has been used as validation for a number of numerical studies, including Hua \& Lou (2007), Lai et al. (2003), Bonometti \& Magnaudet (2007) and Chen et al. (1999). Interestingly, most of the numerical results appear to be in excellent agreement with the experimental observations, except for the formation of unstable toroidal bubbles in systems with large bubbles and low surface tension. Such an instability is not reported by Bhaga \& Weber (1981), where it was observed experimentally that these bubbles tended to form spherical-cap shapes. The reason for the difference experimental and numerical simulation is generally attributed towards the experimental setup, where an inverted hemispherical cup was used to retain a known volume of gas which was slowly rotated to carefully release the bubble within the stagnant liquid. From this procedure the initial shape of the bubble is approximately ellipsoidal; whereas in most numerical studies an initial spherical bubble is assumed. Ohta et al. (2005) have studied the effects of the initial bubble shape on the final state of the bubble, and determined that in cases of high Eotvos $\left(E o=\Delta \rho g D^{2} / \sigma\right)$ and low Morton $\left(M o=\Delta \rho g \mu^{4} / \rho_{l}^{2} \sigma^{3}\right)$ numbers, an initially ellipsoidal bubble (i.e. with its polar axis shorter than the diameter of its equatorial axis) generally assumes a spherical cap bubble shape. Bubbles that are initially spherical or prolate spheroidal in structure, however, tend to develop a toroidal bubble shape. The numerical study of Bonometti \& Magnaudet (2006) is in agreement with Ohta et al. (2005), and suggested a phase diagram to delineate the transition of a spherical bubble to a toroidal shape based on Archimedes and Bond numbers.

Landel et al. (2008) extended the experimental work of Bhaga and Weber (1981) and demonstrated that bubbles released into a column of fluid using an inverted hemispherical cup tend to form a leading spherical cap bubble that is followed by a crown of satellite bubbles ('crowned-cap bubbles'). They reported a dependency on the 
distribution of leading-to-satellite bubble volume on the size of the hemispherical cup, with larger cups tending to produce a larger leading bubble with less satellite bubbles; whereas smaller cups tended to produce a larger volume of satellite bubbles. The conclusion here is that the rise dynamics of large spherical cap bubbles are very much dependent on the initial conditions, although the physical cause of such phenomenon is still largely unknown. It's also noted the initial bubble conditions can determine the onset of non-rectilinear rise behaviour (Wu \& Gharib, 2002).

Whilst significant progress has been in the understanding of the behaviour of gas bubbles rising in a stagnant viscous liquid, there are still many examples (e.g. Cano-Lozano et al., 2013; Zenit \& Magnaudet, 2009; Ellingson \& Risso, 2001; Bonometti \& Magnaudet, 2006; Landel et al., 2008, Tripathi et al., 2015) where the shape, rise velocity, and paths adopted by bubbles, especially in certain Morton, Reynolds and Eotvos number ranges, is not well described numerically. For this reason, in this study a fully $3-\mathrm{D}$, VOF numerical methodology has been employed to determine dynamic shape, drag, mean bubble rise velocity and corresponding liquid flow field for a range of different bubbles in viscous stagnant liquids. Of particular focus is the case for viscous liquids with low surface tension $\left(E O>8\right.$ and $\left.7.4 \times 10^{-4}<M o<850\right)$, where there are noted differences with the experimental observations of Bhaga \& Weber (1981). Reasons for these differences are discussed in the paper.

\section{Numerical methodology}

The simulations are conducted using the Gerris flow solver developed by Popinet (2009). The solver is based on the conservation equations for incompressible flow with variable density and surface tension:

$$
\begin{aligned}
& \nabla \cdot \mathbf{u}=0 \\
& \rho\left(\frac{\partial \mathbf{u}}{\partial \mathbf{t}}+\mathbf{u} \cdot \nabla \mathbf{u}\right)=-\nabla p+\nabla \cdot(2 \mu \mathbf{D})+\sigma \kappa \delta_{s} \mathbf{n},
\end{aligned}
$$

$\mathbf{u}$ is the velocity vector, $\rho$ fluid density, $\mathrm{p}$ pressure, $\mu$ the dynamic viscosity, and $\mathbf{D}$ is the deformation tensor,

$$
D_{i j}=\frac{1}{2}\left(\frac{\partial u_{j}}{\partial i}+\frac{\partial u_{i}}{\partial j}\right) .
$$

$\sigma$ is the surface tension coefficient, $\delta_{s}$ is the Dirac function used to calculate the surface tension force around the interface; $\kappa$ and $\mathbf{n}$ are the curvature and normal vector to the interface.

At each computational cell, the density and viscosity of the fluid are calculated through a volume-of-fluid variable, $\alpha$ :

$$
\rho=\alpha \rho_{1}+(1-\alpha) \rho_{2}
$$




$$
\mu=\alpha \mu_{1}+(1-\alpha) \mu_{2}
$$

$\rho_{1}, \rho_{2}, \mu_{1}$, and $\mu_{2}$ are the density and viscosity of phase 1 and 2 , respectively; $\alpha$ is the volume fraction occupied by phase 1 in a cell, where an $\alpha$ value of 1.0 indicates that the computational cell is filled with the primary phase. The volume fraction variable is advected with the local fluid velocity as follows,

$$
\frac{\partial \alpha}{\partial t}+\nabla \cdot(\alpha \mathbf{u})=0
$$

Staggered temporal discretisation is employed in the solutions of equations 1,2 , and 5 , thus leading to a scheme that is second-order accurate in time (Popinet, 2009). A time-splitting projection method is used to simplify the conservation equations, based on a temporary velocity field, $\mathbf{u}^{*}$, which is calculated through the momentum equation without the effect of pressure. The pressure gradient is then used to calculate the corrected velocity field in the new time step. The pressure field is then obtained from the Poisson equation, i.e.:

$$
\nabla \cdot\left(\frac{1}{\rho} \nabla p\right)=\frac{1}{\Delta t} \nabla \cdot \mathbf{u}^{*}
$$

which is solved using the quad/octree-based multilevel solver (Popinet, 2009), with the iterative procedure being repeated until the maximum volume-weighted residual is smaller than $10^{-5}$, and Gauss-Seidel relaxations per level is set to 6 .

The advection equation for the volume-fraction is calculated through the piecewise linear geometrical Volume-Of-Fluid (VOF) scheme, applied through the quad/octree spatial discretisation. Additionally, the balanced-force surface tension discretization scheme is used, in combination with a height function curvature estimation, such that problems involving parasitic currents normally encountered in VOF-based algorithms using the classical Continuum-Surface-Force (CSF) approach are avoided.

\section{Results and discussion}

\subsection{Computational domain}

A series of simulations were conducted in a $3-\mathrm{D}$ rectangular domain $(9.3 D \times 9.3 D \mathrm{x}$ $27.9 D$ ), where $D$ is the initial diameter of the bubble. Periodic (slip) boundary conditions were used for all of the boundaries of the domain. Adaptive mesh refinement was used, with the maximum level of refinement being applied at the interface between the two phases. The maximum level of refinement, $L_{\max }$, was adjusted based on the predicted Reynolds number of the bubble $\left(R e=\rho V_{t} D / \mu\right.$, where $V_{t}$ is the terminal velocity of the bubble). For $R e \leq 55.3$, a maximum of 9 levels of refinement, corresponding to 55 cells per bubble diameter (CPD), was used at the liquid gas interface with respect to the 
boundary of the domain. Higher orders of refinements were used to resolve cases of skirted bubbles and cases where $R e>55.3$. Further discussions will be presented in the following section regarding the grid dependence of these cases. Lower levels of refinement were used away from the interface; with the minimum level of refinement, $L_{\min }$, being set such that $L_{\min }=L_{\max }-5$. This distribution of mesh ensured that the simulations were computationally stable and efficient. A further refinement scheme based on the velocity magnitude of the cell was employed; where the maximum Reynolds number of the cell, $R e_{\text {cell }}=\rho|V| \Delta x / \mu$, with $\Delta x$ being the size of the cell and $|V|$ the magnitude of the local velocity field, did not exceed 10 .

\subsection{Numerical validation and grid independence}

The simulated shapes of the bubbles after reaching a steady rise velocity are shown in Figure 1. Refer to Table 1 for corresponding numerical values of dimensionless numbers. At low values of Eo and $R e$, it can be seen that the bubbles tend to retain their spherical shape (see Figures 1a and 1b). With higher values of $E o$, the bubbles tend to lose their sphericity, as decreasing influence of surface tension causes the outer rim of the bubble to be drawn-out, thus creating the dimpled (Figures 1g-i), oblate ellipsoidal cap (Figures $1 \mathrm{~d}, \mathrm{j}-\mathrm{l}$ ), and skirted (Figures 1e-f) bubble shapes, which are in agreement with the observations of Bhaga \& Weber (1981). The effects of fluid viscosity and inertia are evidenced in the bubble shapes shown in Figures $1 \mathrm{~g}$-l. The bubble rising in liquid of lower viscosity tends to become flatter due to effects of inertia. Figure 1c shows a flattened disk-like bubble shape, resulting from the inertia due to the buoyancy of the bubble dominating the viscous forces of the fluids. The elongation of the rim of the bubble is resisted, however, due to the high surface tension between the two phases and results in a disk-like bubble shape. 
(a)
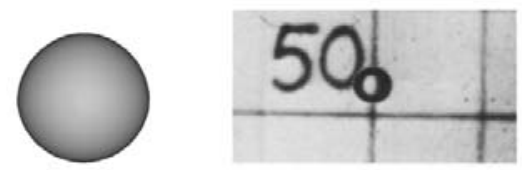

(b)
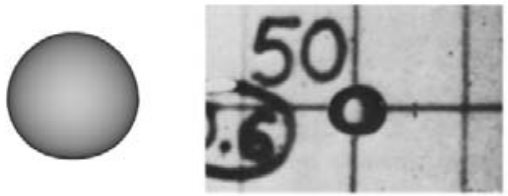

(c)

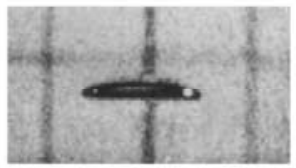

(d)

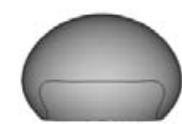

(e)

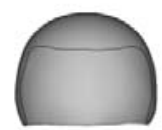

(f)
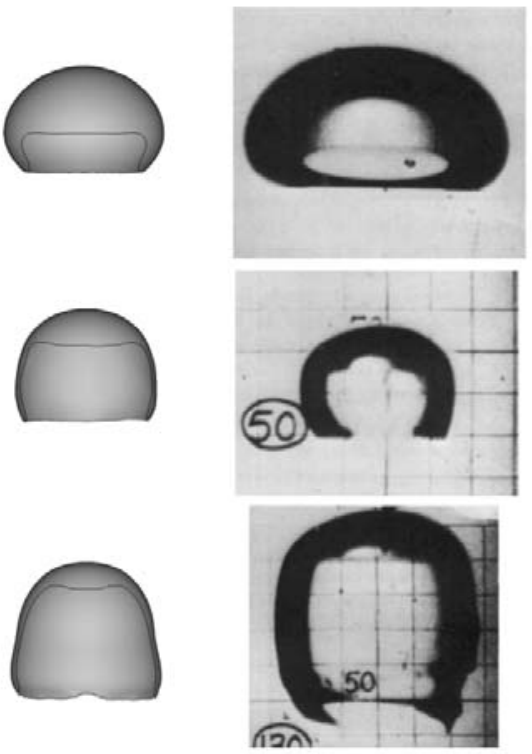

(g)

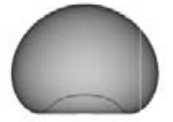

(k)

(h)

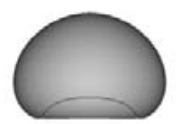

(i)

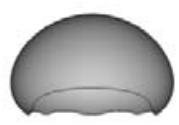

(j)
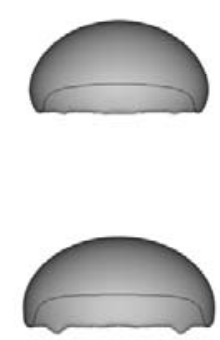

(1)

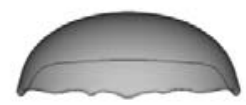

(m)

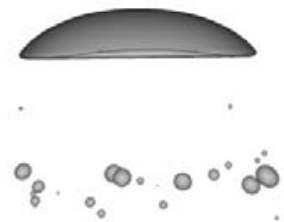

(n)

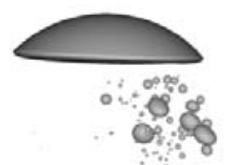

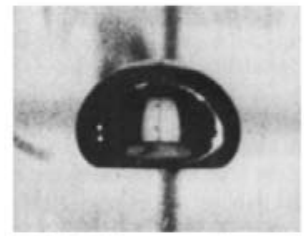
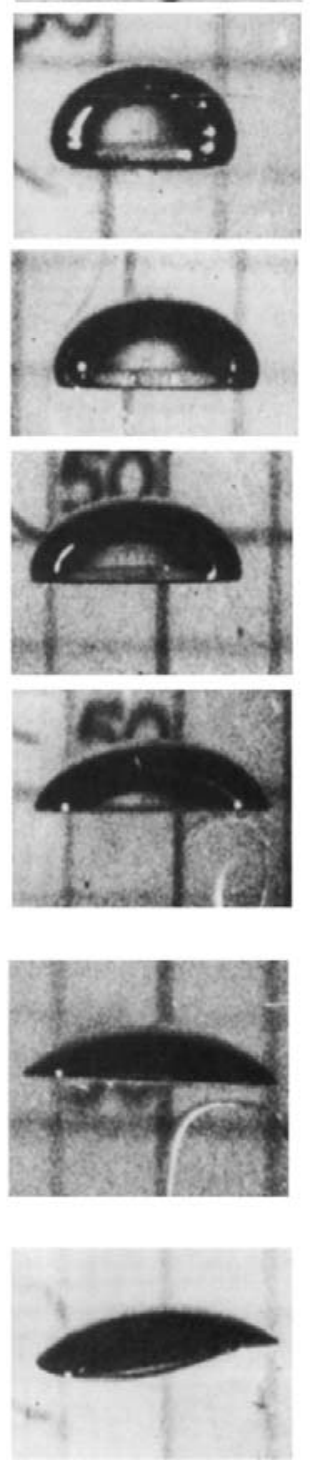

Figure 1. Bubble shapes after reaching constant rise velocity $\left(\rho_{l} / \rho_{g}=1103.7, \sigma=0.07845 \mathrm{~N} / \mathrm{m}\right.$ ) [LHS: Iso-surface and isoline contours at $\alpha=0.5$; RHS: Images from Bhaga \& Weber (1981)] 
Table 1. Bubble dimensionless numbers (see Figure 1)

\begin{tabular}{|c|c|c|c|}
\hline $\begin{array}{c}\text { Figure 1 } \\
\text { reference }\end{array}$ & $E o$ & $M o$ & $\begin{array}{c}R e \\
\text { (Bhaga and Weber, 1981) }\end{array}$ \\
\hline (a) & 8.67 & 711 & 0.078 \\
\hline (b) & 17.7 & 711 & 0.232 \\
\hline (c) & 32.2 & $8.2 \times 10^{-4}$ & 55.3 \\
\hline (d) & 243 & 266 & 7.77 \\
\hline (e) & 339 & 43.1 & 18.3 \\
\hline (f) & 641 & 43.1 & 30.3 \\
\hline (g) & 115 & 848 & 2.47 \\
\hline (h) & 115 & 266 & 3.57 \\
\hline (i) & 115 & 41.1 & 7.16 \\
\hline (j) & 115 & 5.51 & 13.3 \\
\hline (k) & 115 & 1.31 & 20.4 \\
\hline (l) & 115 & 0.104 & 42.2 \\
\hline (m) & 115 & 0.005 & 94.0 \\
\hline (n) & 115 & 0.001 & 151.0 \\
\hline
\end{tabular}

The relatively good agreement in computed in bubble shape with those reported by Bhaga \& Weber (1981) at $R e \leq 55.3$ is further evidenced by comparison in the respective bubble rise velocities as shown in Figure 2. Good agreement was obtained, especially at low values of bubble Reynolds numbers $(R e \leq 20)$. At higher Reynolds numbers, boundary layer separation tends to develop in the wake of the bubble, thus causing an increase in drag and a decrease in velocity. Consequently, the bubbles would firstly attain a maximum velocity, before decreasing towards a (lower) terminal velocity as demonstrated by the instantaneous velocity graphs shown in Figures $2 \mathrm{~b}-2 \mathrm{~d}$. The time variance of the simulated bubble velocity is quantified by the vertical error bars. Given that Bhaga \& Weber (1981) determined the velocity of the bubbles by calculating the distance travelled by a particular bubble in two adjacent frames and dividing the distance with the time elapsed, the agreement shown in Figure $2 \mathrm{a}$ is an excellent result. 


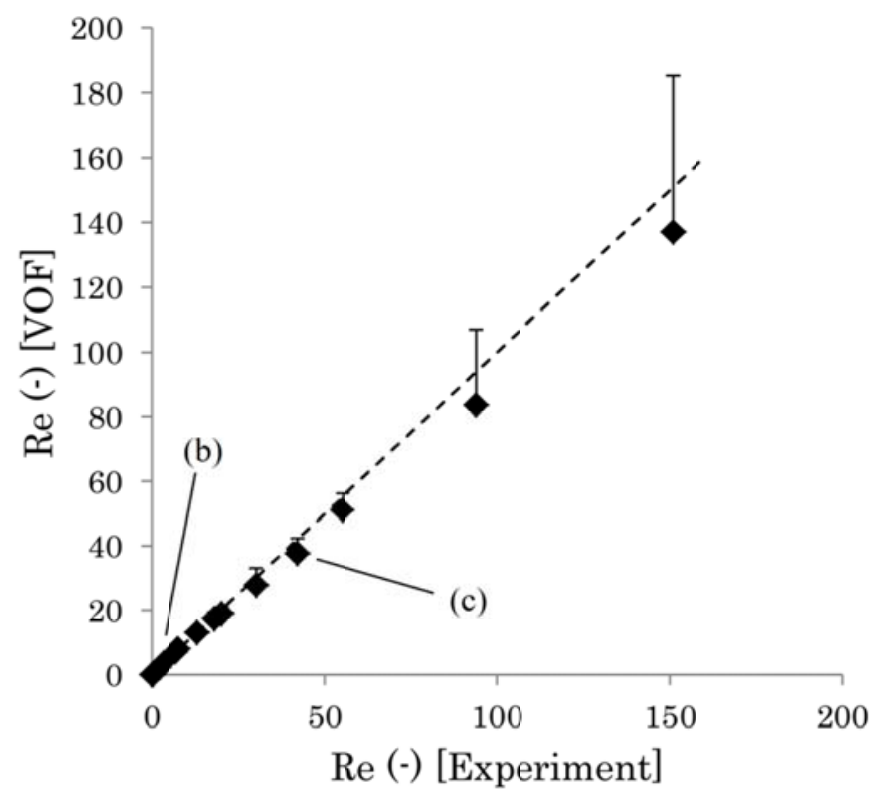

Figure 2a. Simulated vs Bubble Reynolds number reported by Bhaga \& Weber (1981) [Points (b) and (c): Bubble rise velocity and fluid streamlines shown in Figures 2b and 2c]

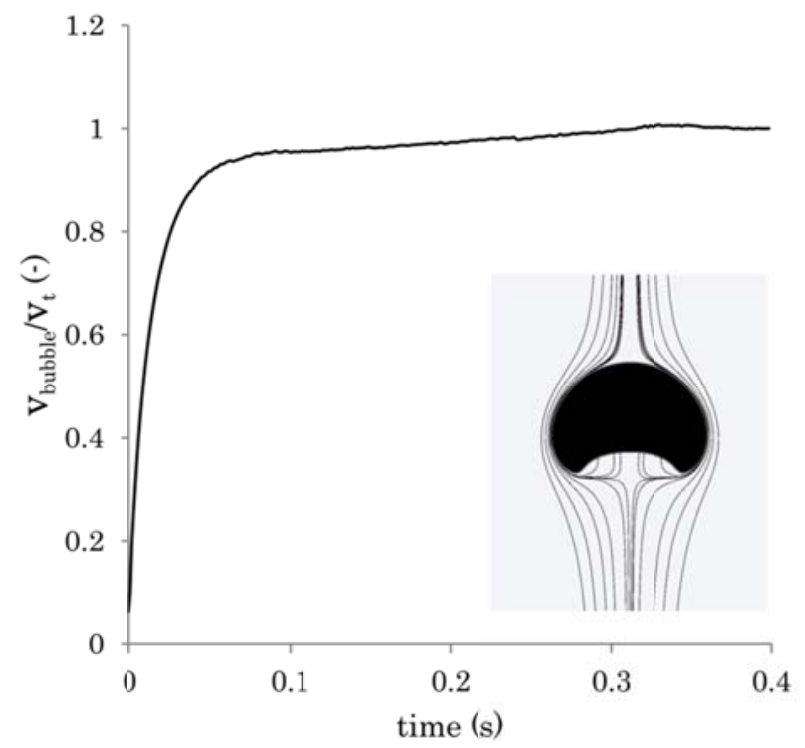

Figure $2 \mathrm{~b}$. Bubble rise velocity and vs time and liquid streamline at $\mathrm{t}=0.4 \mathrm{~s}$ [Point b: $R e=3.57 E o=116, M o=266$ ] 


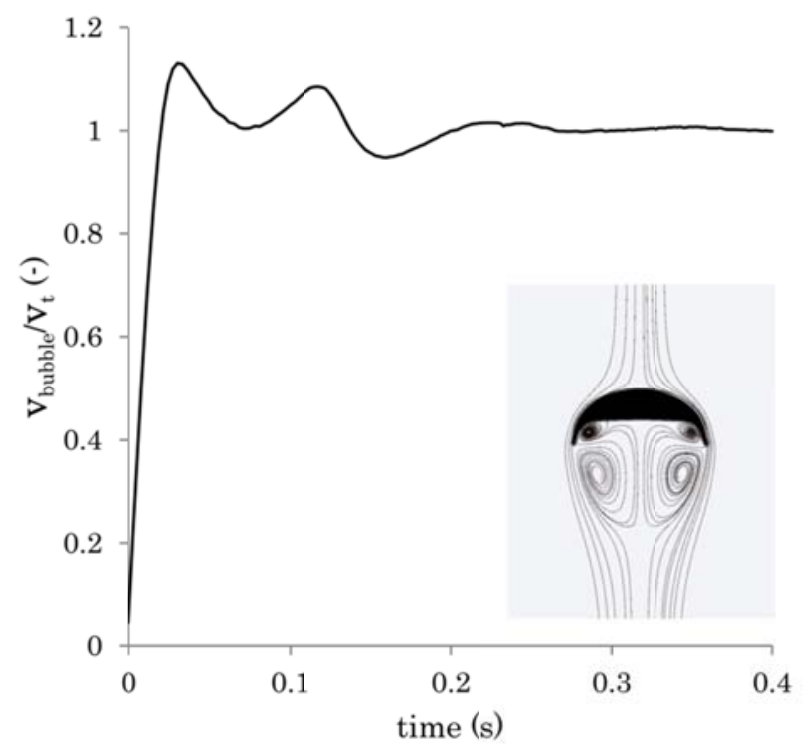

Figure 2c. Bubble rise velocity and vs time and liquid streamline at $\mathrm{t}=0.4 \mathrm{~s}$ [Point b: $R e=42.2, E o=116, M o=0.103$ ]

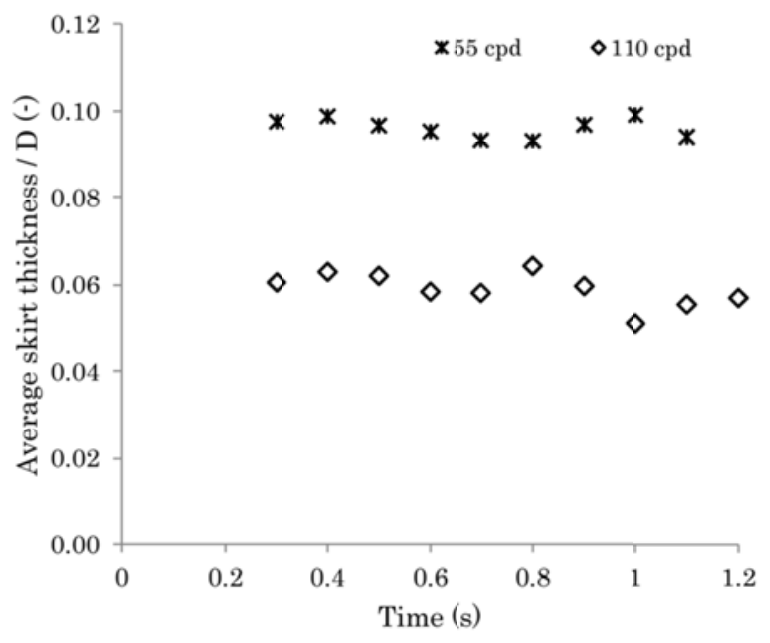

Figure 3. Average skirt thickness as a function of time [Refer to Figure 1f: $E o=339, M o=43.1, R e=18.3$ ]

The behaviour of bubbles at higher values of $R e$ is generally complicated by the formation of thin and fragmented segments, which require higher levels of refinement for their resolution. This is especially true for the formation of skirted bubbles. Figure 3 shows the development of the skirt segment of a bubble rising at $E o=339$ and $R e=18.3$. The average thickness of the skirt was calculated by $\bar{\delta}=\int_{y_{0}}^{y_{L}} \delta \partial y / \int_{y_{0}}^{y_{L}} \partial y$, where $\delta$ is the thickness of the skirt at point $\mathrm{y}$, and $y_{L}-y_{0}$ is the length of the skirt section. The thickness of the skirt produced by the $110 \mathrm{cpd}\left(L_{\max }=10\right)$ simulation is $\approx 40 \%$ lower 
than that of the $55 \mathrm{cpd}\left(L_{\max }=9\right)$ simulation. This indicates that the simulations of the skirt regions of the bubbles cannot be considered grid independent. In comparison to the work of Wairegi (1974), the average thickness of the skirt based on the $110 \mathrm{cpd}$ simulations is found to be considerably higher ( $\sim 50$ times $)$ than the predicted value,

$$
\delta=\sqrt{\frac{6 \mu_{\text {bubble }} V_{t}}{\left(\rho_{l}-\rho_{\text {bubble }}\right) g}} .
$$

Equation 7 is derived based on the balance between the pressure gradient in the skirt along the vertical direction with the pressure gradient outside the boundary layer of the host liquid (Ray and Prosperetti, 2014). Ohta and Sussman (2012) have performed a set of 2-D simulation studies on skirted bubbles and drops using the Coupled Level Set Volume of Fluid (CLSVOF) method, and found a dependency in the average thickness of skirt formations on the density $\left(\rho_{\text {gas }} / \rho_{\text {liquid }}\right)$ and viscosity $\left(\mu_{\text {gas }} / \mu_{\text {liquid }}\right)$ ratios of the discrete and continuous phases. The thickness of the skirt was found to decrease with decreasing density and viscosity ratios. Resolution levels of up to $1024 \mathrm{cpd}$ were used to analyse the thickness and length of the skirt section. Their results indicated that at the typical density and viscosity ratios of gas-liquid systems, a bubble rising at $E O=339$ and $R e=18.3$ would form a very thin trailing skirt, which, even with $1024 \mathrm{cpd}$ resolution formed a skirt that is less than one-grid cell thick. In 3-D simulations, such level of grid resolution is highly unfeasible with the current level of computing power; accurate analysis of skirted bubbles at high Reynolds numbers is therefore an area in need of further research, requiring a greater understanding on the spatial discretisation of the skirt segment.

Despite the differences in the thicknesses of the skirts formed in the 55 and $110 \mathrm{cpd}$ simulations, the terminal velocities of the bubbles are found to be relatively uniform $(0.373 \mathrm{~m} / \mathrm{s}$ and $0.394 \mathrm{~m} / \mathrm{s}$ for the 55 and $110 \mathrm{cpd}$ simulations, respectively). This is consistent with the findings of Ohta and Sussman (2012), who found that the thickness of the trailing skirt have little effect on the rise velocity of the bubble. This indicates that while the current level of grid refinement is inadequate in resolving the skirt region of the bubbles, it is able to yield a representative description of the flow fields surrounding them. For the current study, resolution level of $110 \mathrm{cpd}$ is used to simulate skirted bubbles cases.

The formation of satellite bubbles evident in Figure $1 \mathrm{~m}$ results from the development of a trailing skirt behind the rising bubble. As the trailing skirt gets pulled along, its thickness tends to decrease, eventually breaking apart and forming detached bubbles in the wake of the larger bubble. The formation of satellite bubbles in the wake is therefore also dependent on the resolution of the trailing skirt. As in the case with the skirted bubbles, the $110 \mathrm{cpd}$ simulation is found to produce a thinner trailing skirt than the 55 cpd simulation, thus resulting in a lower rate of satellite bubble production. At $0.4 \mathrm{~s}$ from the start of the simulation, the $110 \mathrm{cpd}$ bubble is found to produce $\approx 2.3 \%$ satellite bubbles with respect to the original volume of the bubble. On the other hand, the $55 \mathrm{cpd}$ bubble sheds $7.4 \%$ of its original volume within $0.4 \mathrm{~s}$ from the start of the simulation. This results in a slight discrepancy in the bubble terminal velocity; the $55 \mathrm{cpd}$ 
simulation produces a bubble terminal velocity that is $\approx 5 \%$ lower than the $110 \mathrm{cpd}$ (the $110 \mathrm{cpd}$ simulation produces terminal velocity of $0.319 \mathrm{~m} / \mathrm{s}$, or $R e=83.8, \approx 10.8 \%$ lower than the reported terminal velocity value of Bhaga and Weber (1981)). The maximum velocity reached by the bubble in both cases is $0.408 \mathrm{~m} / \mathrm{s}$, corresponding to $R e_{\max }$ of 107.1. The range of Reynolds number attained by the simulations therefore still tends to fall within the reported value of Bhaga \& Weber (1981) (see Table 1, Figures $1 \mathrm{~m}$ and 2), despite the uncertainties involved with the production rate of the satellite bubbles.

Simulations of large bubbles $(R e>100, E O>100)$ that are initially spherical in shape generally result in a toroidal bubble formation (Chen et al., 1999; Ohta et al., 2005; Bonometti \& Magnaudet, 2006; Hua and Lou, 2007; Tripathi et al., 2015). This is in contrast to the observations of Bhaga \& Weber, who reported the formation of spherical cap bubbles with open wakes in the region of $151 \leq R e \leq 259$ and $115 \leq E o \leq 237$. The discrepancies appear to be largely caused by the initial shape of the bubble: those that are initially spherical in shape tend to inhibit a toroidal bubble formation, whereas bubbles that are initially ellipsoidal in shape tend to inhibit a spherical cap bubble formation (Ohta et al., 2005). Indeed, due to the experimental setup employed by Bhaga \& Weber, wherein the bubble is initially contained in an inverted hemispherical cup, which is then turned slowly to release the bubble into the column of fluid, the initial shape of large bubbles may have been rendered ellipsoidal upon release.

The findings of Ohta et al. (2005) were further supported by the study of Bonometti \& Magnaudet, who suggested the presence of stable and unstable regions, in which the final shape of an initially spherical bubble rising at large $E O$ and $R e$ values could either be spherical cap or toroidal, depending on the viscous and capillary contributions of the fluid properties. This was further analysed by Tripathi et al. (2015), who suggested a slightly different delineation for stable and unstable regions based on their 3-D simulation results. Based on these stability criteria suggested by both Bonometti \& Magnaudet (2006) and Tripathi et al. (2015), the parameters used in the case of $E O=$ 115 and $R e=151.0$ fall within the unstable region; it is therefore expected that the bubbles attain toroidal bubble formation throughout all levels of refinement. As can be seen in Figure 4, simulations with lower levels of resolution (55 and $110 \mathrm{cpd}$ ) indeed show this behaviour, with the bubble developing a stable toroidal formation after 0.08-0.10 s. However, in the case of the $220 \mathrm{cpd}$ simulation, the bubble tends to only temporarily adopt a semi-toroidal structure, before re-forming the top layer and reverting into a spherical cap structure. The 3-D shape of the bubble based on the 220 cpd simulation, $0.67 \mathrm{~s}$ from stationary can be seen in Figure 1n. The terminal velocity of the bubble based on the $220 \mathrm{cpd}$ was $0.338 \mathrm{~m} / \mathrm{s}, \approx 9 \%$ lower than the reported value of Bhaga and Weber (1981). Similar to previous cases of flow separation, some fluctuation occurs in the rise velocity of the bubble. The maximum velocity achieved by the bubble during its rise from rest is $0.457 \mathrm{~m} / \mathrm{s}$, thus corresponding to $R e_{\max }$ of 185.2 , indicating that the range of Reynolds numbers produced by the $220 \mathrm{cpd}$ simulation encompasses the Reynolds number reported by Bhaga and Weber (1981) (see Table 1, Figures 1n and 2 ). The asymmetry of the bubble, as evident in Figure 1n, suggests the formation of an open wake; this will be discussed in the following sections. 
$0 \mathrm{~s}$

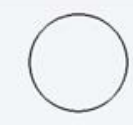

$0.05 \mathrm{~s}$

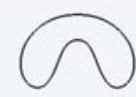

$0.07 \mathrm{~s}$

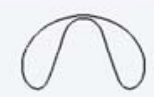

$0.08 \mathrm{~s}$
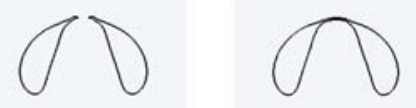

$0.09 \mathrm{~s}$
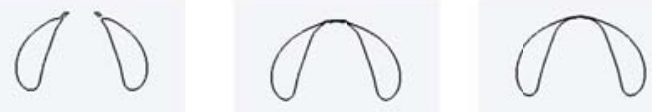

$0.10 \mathrm{~s}$
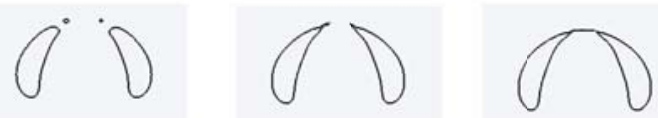

$0.11 \mathrm{~s}$
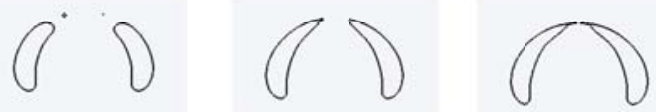

$0.12 \mathrm{~s}$
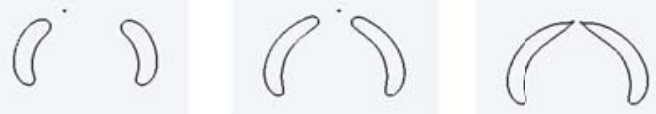

$0.13 \mathrm{~s} \quad(2)$<smiles>c1ccccc1</smiles><smiles>c1ccccc1</smiles>

$0.14 \mathrm{~s} \quad(2)$<smiles>c1ccccc1</smiles><smiles>c1ccccc1</smiles>

$0.16 \mathrm{~s}$
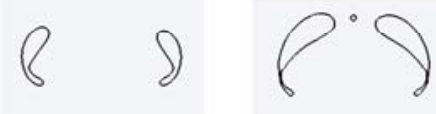

$110 \mathrm{cpd}$
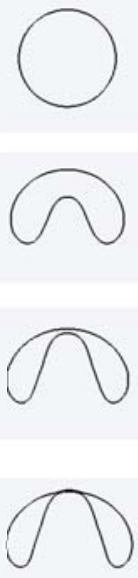

$55 \mathrm{cpd}$

Figure 4. Isoline contours at $\alpha=0.5$ of a bubble rising at $E o=115, M o=0.001, R e=151.0$ at different resolution levels. 

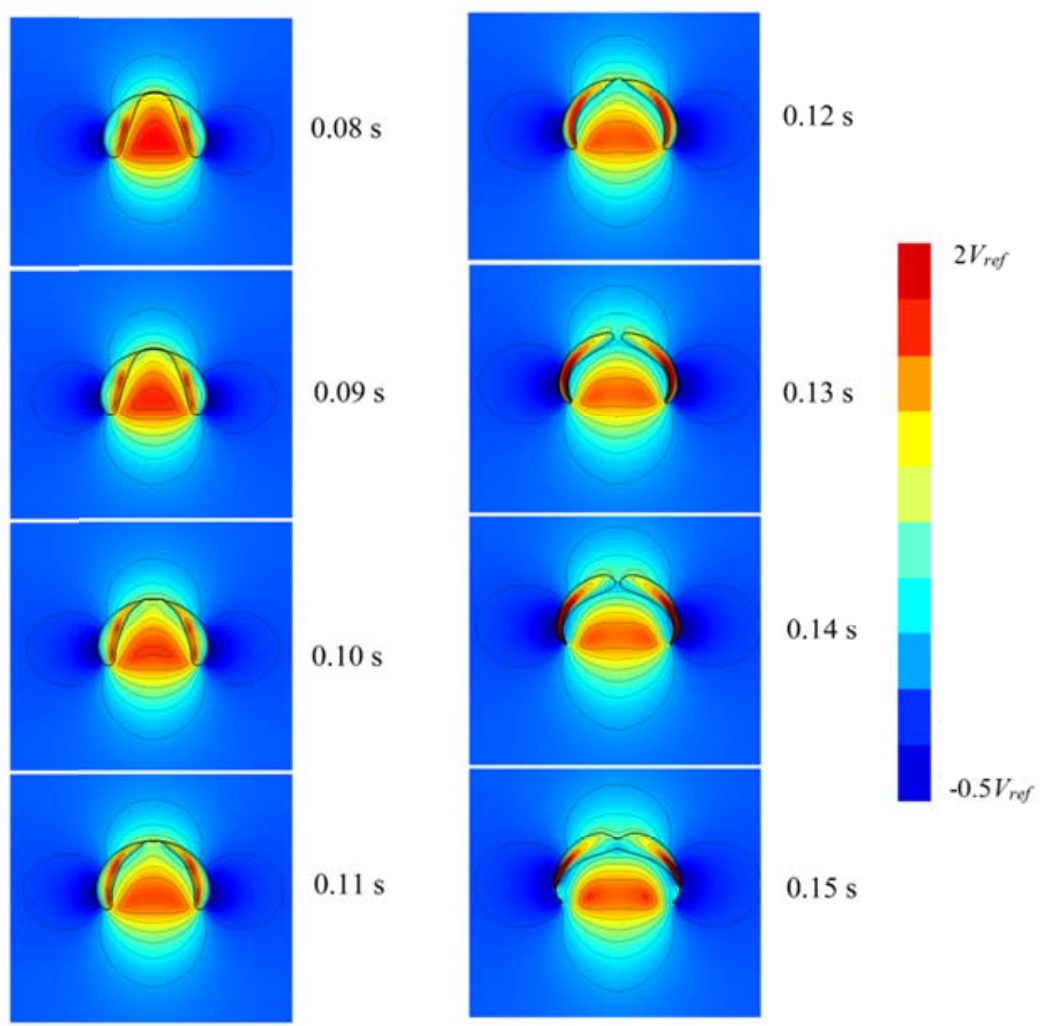

Figure 5a. Contour of vertical velocity $\left(R e=151, M o=1 \times 10^{-3}, E o=115\right)$ normalised against $\mathrm{V}_{\text {ref }}$ [Bubble velocity calculated based on the reported value of Reynolds number of Bhaga \& Weber (1981)] 


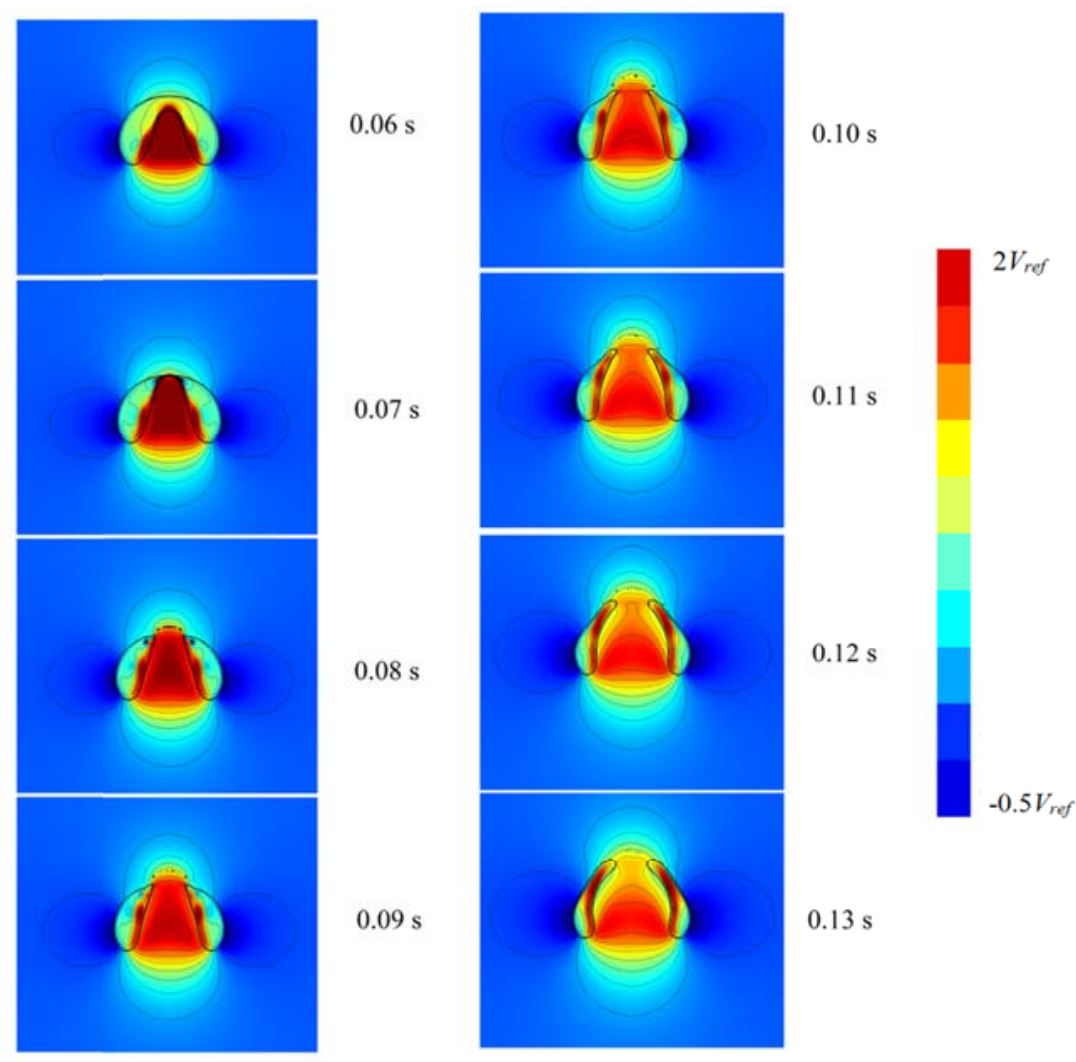

Figure 5b. Contour of vertical velocity $\left(R e=259, M o=1 \times 10^{-3}, E o=237\right)$, normalised against $\mathrm{V}_{\text {ref }}$

The formation of the toroidal structure is driven by the development of a very thin film at the centre of the leading edge of the bubble. The mechanism of this formation has previously been studied by Walters \& Davidson (1963), Chen et al. (1999), and Bonometti and Magnaudet (2006). It was found that the lower surface of large bubbles rising in liquids tend to undergo significant deformation due to the presence of a upward liquid jet in the wake, which then pierces the top surface. The development of the liquid jet for two cases presented by Bhaga \& Weber (1981) can be seen in Figures 5a and b. The first case (Figure 5a) is essentially the one presented in Figures $1 \mathrm{n}$ and $4(R e=151$, $\left.M o=1 \times 10^{-3}, E o=115\right)$, whereas the second case has higher values of $R e$ and $E o$ numbers $\left(R e=259, M o=1 \times 10^{-3}, E o=237\right)$. In both cases, maximum resolution levels of $220 \mathrm{cpd}$ were used. Both Figures $5 \mathrm{a}$ and $5 \mathrm{~b}$ show that as the top surface of the bubble is pierced, the jet becomes wider, and its intensity decreases somewhat due to the increase in surface area. In the case presented in Figure 5a, the decrease in the intensity of the jet within the bubble torus, combined with the effect of fluid recirculation at the outer layer of the bubble, is sufficient to create a net force that pushes the top layer of the bubble back inwards, thus reforming a spherical cap shape. On the other hand, the jet formation in the case presented in Figure $5 \mathrm{~b}$ is considerably more prominent, i.e. the average velocity in the jet is much higher in comparison to the bubble rise velocity. Upon rupture, the intense kinetic energy of the jet tends to push the sides of the torus apart, therefore causing the bubble toroid to widen. 
The formation of toroidal bubbles, as shown in Figure 5b, therefore depends on the intensity of kinetic energy of a liquid jet that is formed at the wake of the bubble, and the recirculation of fluid surrounding it. Further, the formation of an open turbulent wake as evident in Figure 1n (to be discussed in the following section), which tends to weaken the upward jet and thus stabilises the spherical cap bubble shape, could not have been captured with the 2-D simulations performed by Bonometti and Magnaudet (2006) and Ohta et al. (2005). These findings highlight the importance of 3-D flow field and the spatial resolution in the simulation of high $R e$ and Eo bubbles.

The results presented in this section demonstrate thoroughly the validity of the current numerical methodology in representing the various shape regimes of bubbles rising in viscous liquids. However, some limitations remain, especially in the representation of thin skirt segments of skirted bubbles. As a result, the formation of satellite bubbles in the wake of a leading bubble also cannot be represented with good accuracy. Nevertheless, the excellent agreement of the rise velocity of the bubbles with the experimental observations of Bhaga and Weber (1981) indicate good representation of the flow fields around the bubbles have been obtained.

\subsection{Bubble aspect ratio}

The shape of the bubbles presented in Table 1 can be seen to generally fall into 6 categories: spherical (Figure 1a), oblate ellipsoidal (Figure 1b), oblate ellipsoidal disk (Figure 1c), oblate ellipsoidal cap (Figures 1d, g-l), skirted (Figures 1e-f) and spherical cap (Figures $1 \mathrm{~m}^{-n}$ ). In this section, the dependency of the bubble aspect ratio on the parameters of the liquid and gas phases will be analysed.

In the case of the spherical and ellipsoidal bubbles (Figures 1a and b), the aspect ratio [ $\chi=$ minor (vertical) axis / major (horizontal) axis] of the bubbles is found to be relatively close to 1.0 ( $\chi=0.97$ and 0.95 respectively for bubbles of Figures $1 \mathrm{a}$ and $1 \mathrm{~b}$ ). The boundary layer stays attached to the surface of the bubble, maintaining fore-and-aft symmetry in the velocity flow field.

The fore-and-aft symmetry observed in the shapes of the spherical and ellipsoidal bubbles is absent for cases of oblate ellipsoidal cap bubbles (Figures 1d, 1g-1). This is reflected in the flow fields around the bubbles, in which liquid particles at the rear of the bubbles have to move along the rim before progressing downstream. In the case of the bubble presented in Figure 1g $(\chi=0.76)$, the inertia within the flow field is sufficiently low such that liquid at the rear of the bubble could move along the bubble interface without separating away from it. Boundary layer separation starts to occur in cases with slightly higher values of $R e$, i.e. in the cases presented in Figures $1 \mathrm{~d}$ and $1 \mathrm{~h}-\mathrm{i}(\chi=0.64$, 0.70 , and 0.63 , respectively, with $3.57 \leq R e \leq 7.77$ ). However, the separation in these cases does not induce any further flow irregularities downstream from the bubble surface (see Figure $2 \mathrm{~b}$ ). In cases with higher values of $R e(13.3 \leq R e \leq 44.2)$, boundary layer separation at the rear of the bubbles causes the formation of a toroidal wake. Additionally, an area of re-circulation just behind the rim of the bubble could also be 
seen to form at even higher values of $R e$, notably at $R e \geq 20.4$ and $\chi<0.47$ (see Figure $2 \mathrm{c}$ for the wake formation of the bubble presented in Figure 11).

In the case of oblate ellipsoidal disk bubbles (Figure 1c), strong fore-and-aft asymmetry in the shape of the bubble induces flow separation that leads to the formation of a closed toroidal wake, similar to the case of the oblate ellipsoidal cap bubbles.

Legendre (2012) has proposed a correlation for the aspect ratio of spherical and ellipsoidal bubbles based on their experimental observations:

$$
\chi=1-9 / 64 \mathrm{We}\left(1+0.2 \mathrm{Mo}^{0.1} \mathrm{We}\right)^{-1}
$$

where $W e$ is the Weber number, We $=\rho_{l} V_{t}^{2} D / \sigma$. The agreement of this correlation with the bubble cases presented in Table 1 can be seen in Figure 6. The data has been classified into two groups: (A) cases of bubbles without a standing eddy and (B) cases of bubbles with a standing eddy, including skirted and spherical cap bubbles. It can be seen that the correlation generally provides good prediction of the aspect ratio of the bubbles as a function of $W e$ and $M o$ numbers, except for cases where the bubbles exhibit spherical cap or skirted bubble formations. The errors associated with case A bubbles, i.e. those without a standing eddy or toroidal wake formations, are relatively low $(<10 \%)$, whereas the prediction of the bubble aspect ratio for case B bubbles involve greater levels of uncertainties (10-46\%). The correlation was found to fail in cases of spherical cap and skirted bubbles.

Figure 6 shows an excellent linear fit between the two sets of simulation results with the results of equation 8 (this was obtained through considerations of all data, with the exception of spherical cap and skirted bubble formations), although a much better fit was clearly shown for cases where non-toroidal wake formation is obtained. 


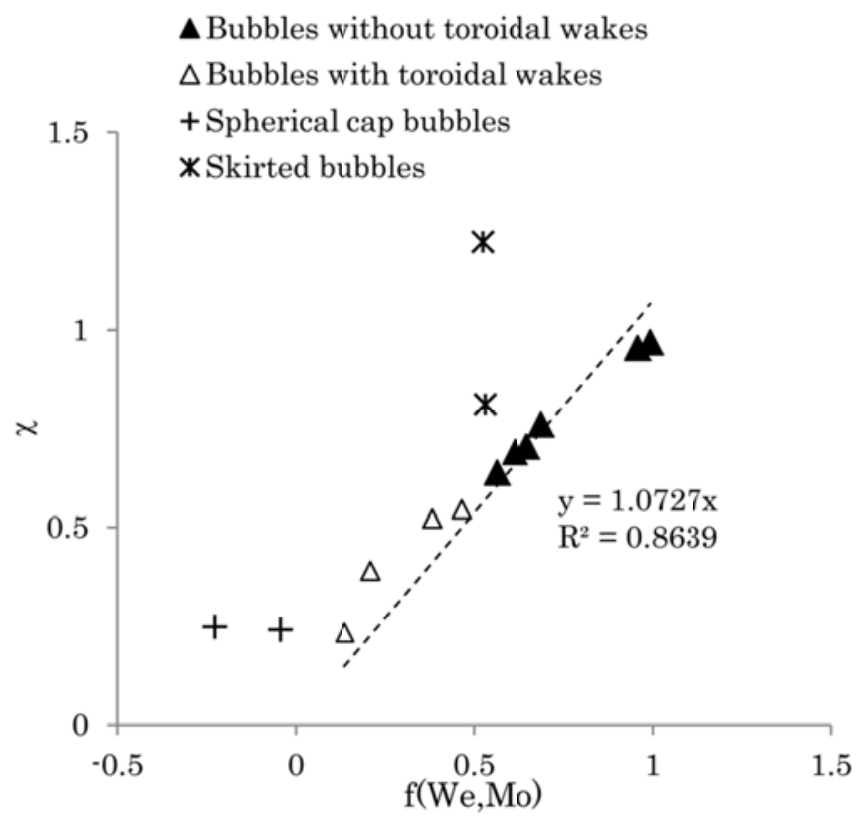

Figure 6. Bubble aspect ratio ( $\chi=$ minor (vertical) axis/major (horizontal) axis) in comparison to the correlation of Legendre et al. (2012) (see equation 8):

(a) bubbles without toroidal wake formation; (b) bubbles with toroidal wake formation.

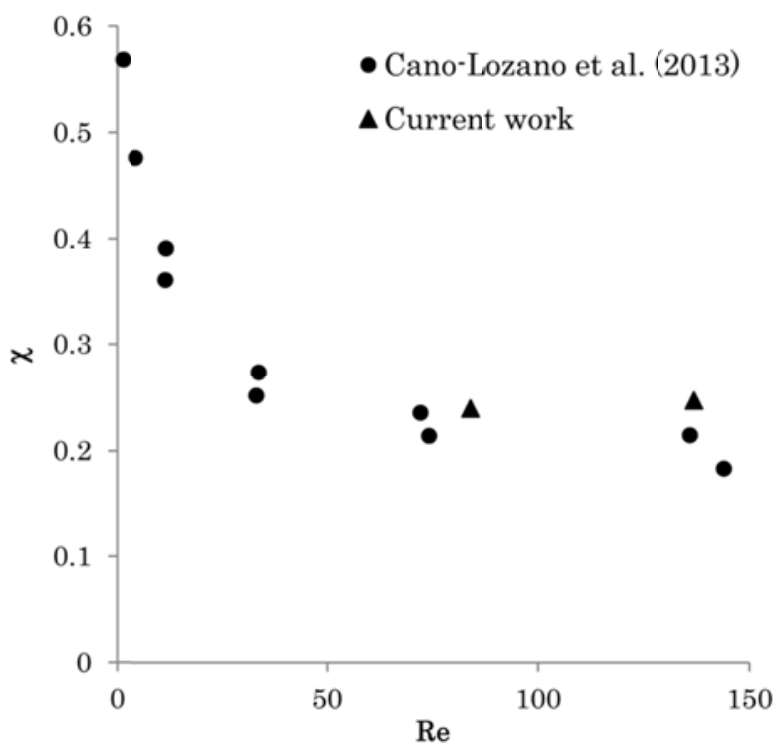

Figure 7. The aspect ratios of spherical cap bubbles as a function of Reynolds number.

The discrepancy in the bubble aspect ratio values in the case of spherical cap bubbles in comparison to the correlation suggested by Legendre et al. (2012) may have been caused by the strong dependency of the aspect ratio in bubbles of these cases to the $R e$ values. Figure 7 shows the aspect ratio of spherical cap bubble cases as a function of $R e$; a generally strong decreasing trend of the aspect ratio with increasing values of $R e$ is 
evident. This is in agreement with the results of Bhaga and Weber (1981), where a correlation between the bubble aspect ratio and its Reynolds number has been suggested.

On the other hand, the deviation of the cases of skirted bubbles from the predicted values of aspect ratio is caused by the length of the bubble skirt, which has not been taken into account in equation (8). The dimensions of the skirt segment of skirted bubbles and drops have previously been studied by Ray and Prosperetti (2014). The length of the skirt, $L=y_{L}-y_{0}$, where $y_{0}$ is the lowest point of the bubble at its line of symmetry and $y_{L}$ is the lowest point of the skirt, is made dimensionless through considerations of the gravitational acceleration and the rise velocity of the bubble, $L^{*}=g L / V_{t}^{2}$. Ray and Prosperetti postulated that $L^{*}$ is a function of the combination of the Capillary and Eotvos numbers, $\mathrm{Ca} / \mathrm{EO}^{0.5}$, as well as the viscosity and density ratios of the two phases. The Capillary number is defined as a ratio between viscous and surface tension forces,

$$
C a=\mu_{l} V_{t} / \sigma .
$$

The dependency of the dimensionless skirt length as a function of $\mathrm{Ca}$ and the viscosity ratio $\left(\mu_{d} / \mu_{c}\right.$, where $\mu_{d}$ and $\mu_{c}$ are the viscosities of the dispersed and continuous phases, respectively), for cases of skirted bubbles in the current study (cases e-f in Figure 1 and Table 1) can be seen in Figure 7. In combination with this data, the results presented by Wairegi (1974) on the length of skirted droplets in sucrose solutions have been included. The complete data, which comprises of $115 \leq E O \leq 717$, and density ratios of $9.06 \times 10^{-4} \leq \rho_{d} / \rho_{c} \leq 1.15$, shows that a strong linear correlation occurs between $L^{*}$ and $C a$.

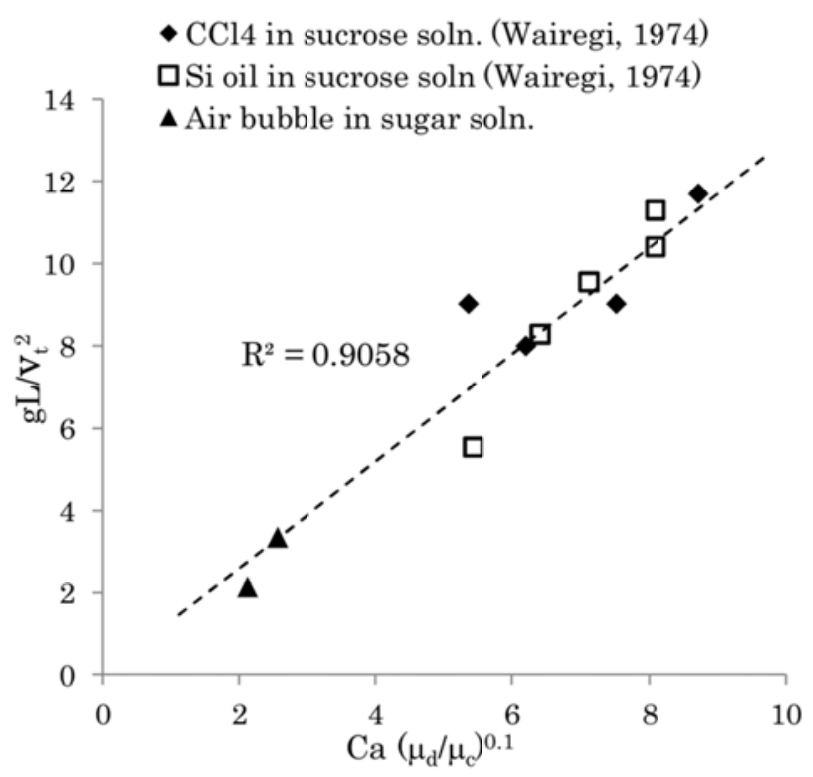

Figure 8. Dimensionless parameter of skirt length as a function of the Capillary number. 
The findings in this section suggest that the aspect ratio of bubbles in different regimes of bubble shape is dependent on a number of different dimensionless parameters. In the case of spherical and ellipsoidal bubbles, strong dependency is demonstrated with the Morton and Weber number, indicating that it is largely driven by the surface tension between the two phases. In the case of spherical cap bubbles, inertia and viscous forces tend to dominate, and a strong dependency of the bubble aspect ratio on the bubble Reynolds number is demonstrated. Finally, in the case of skirted bubbles, strong dependency was found to occur between the length of the skirt on the surface tension and viscosity of the two phases. The aspect ratio of these bubbles is therefore expected to be a strong function of the Capillary number of the system.

\subsection{Wake characteristics and liquid-induced velocity}

In the simulation results presented in Figure 1, except for cases where $R e<2.5$ and cases with the high Reynolds number $(R e>55.3)$, the formation of laminar and hydrodynamically stable toroidal wakes are obtained, with a toroidal vortex ring formed inside them. Inside the wake, the axial velocity of the liquid phase increases away from the gas-liquid interface, before decreasing steadily towards zero. This can be seen in Figure 9a, where variation of the vertical component of the velocity vector along the vertical axis of symmetry of the bubble has been presented for two different cases: the oblate ellipsoidal cap bubble presented in Figure 1l (Figure 2c for streamline) and the skirted bubble presented in Figure 1f (Figure 9b for streamline). In the case of the skirted bubble, the velocity profile within the primary wake is slightly different, mainly due to the presence of a large circulatory region close to the rim of the bubble (see Figure 9b). While this is also observed in the case of oblate ellipsoidal cap bubbles rising at $R e \geq 20.4$ and $\chi<0.47$ (see Figure 2c), the size of the recirculation area in the case of the skirted bubble appears to be significantly larger. As a result, the axial velocity in the liquid phase tends to decrease away from the surface of the bubble, before increasing towards a maximum value at $70 \%$ of the length of the primary wake, $L_{W}$. 


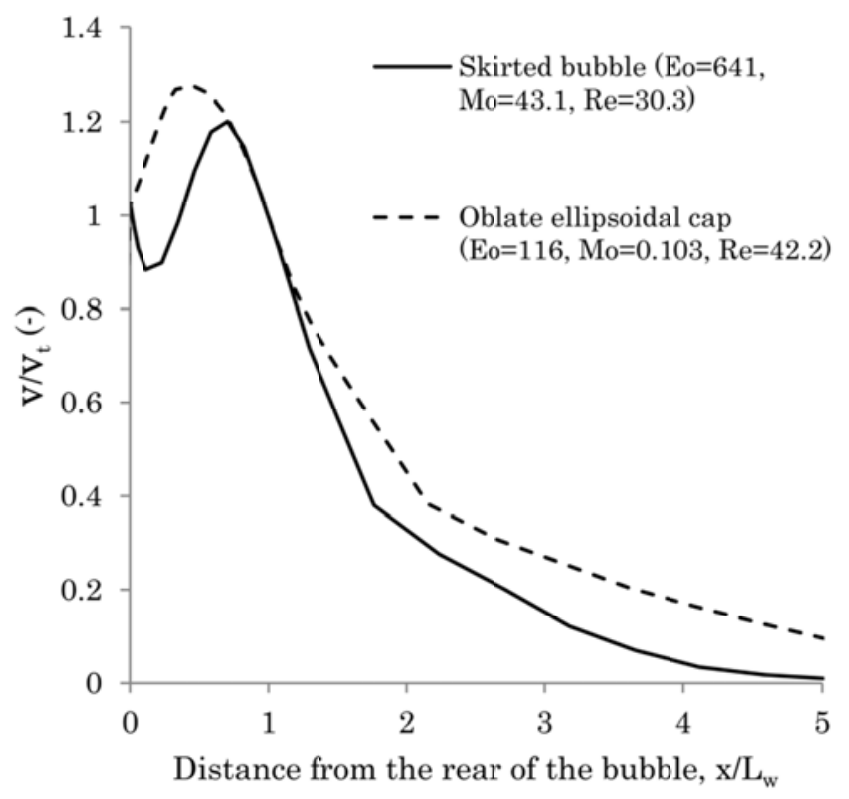

Figure 9a. Vertical velocities in the liquid along the vertical axis of symmetry of the bubble. Refer to Figure $9 \mathrm{~b}$ for the notation of the axes.

The extension of the primary wake can be estimated by calculating the distance from the lowest point of the bubble at its line of symmetry to the point at which the vertical velocity is equal to the rise velocity of the bubble. Except for cases with high values of $R e$ ( $R e \geq 55.3$ - these cases will be discussed later in this section), the extension of the primary wakes is found to attain steady state values within $0.4-0.8 \mathrm{~s}$.

The presence of toroidal wakes behind a rising bubble has previously been examined by Cano-Lozano et al. (2013), who presented a critical curve of Galileo number as a function of the Bond ( $\approx$ Eotvos) number. Above this curve, a recirculating (or toroidal) wake is expected to occur, and vice versa. In the current work, this critical curve has been translated into an Eotvos-Reynolds number curve. Additionally, data based on the observations obtained so far at $8.67 \leq E O \leq 641$ on the presence (or absence) of toroidal wakes has been incorporated, by estimating the critical values of $R e$ at different values of $E o$ in this range. The resulting curve of critical Reynolds number can be seen in Figure 10a, where it is evident that the critical value of Reynolds number can be correlated with the Eotvos number through a power function. 


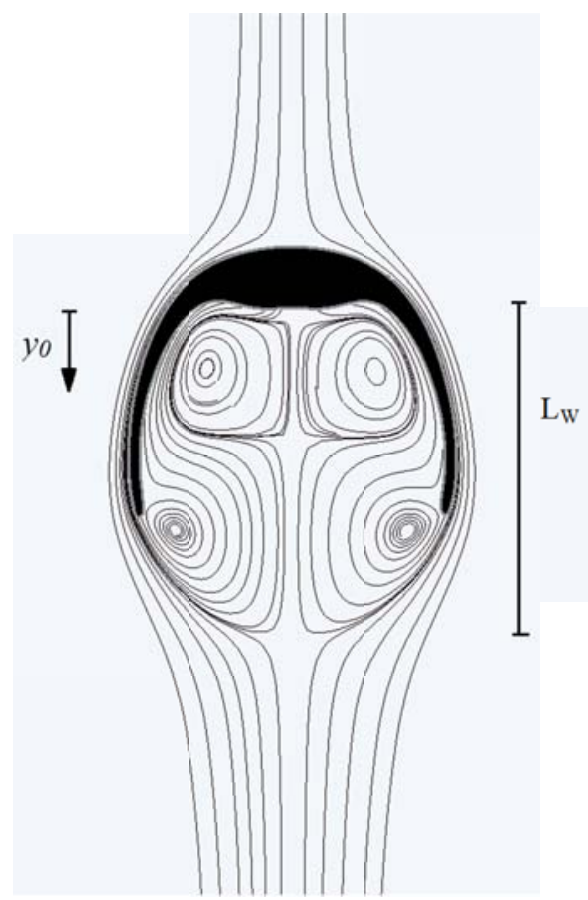

Figure 9b. Streamline of the velocity flow field [Eo=641, $M o=43.1, R e=30.3]$.

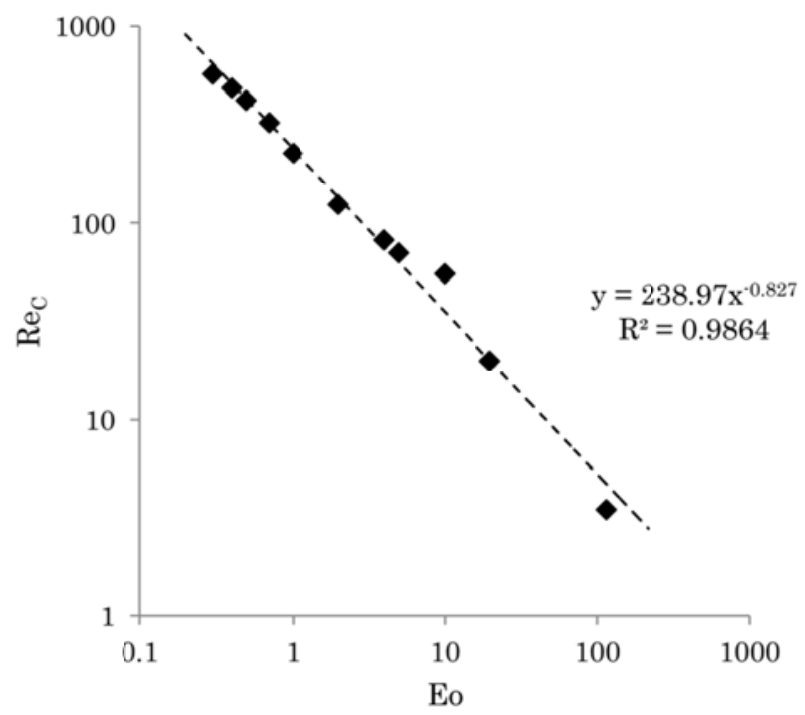

Figure 10a. The critical Reynolds number, as a function of the Eotvos number, above which toroidal wake formation is expected, and vice versa.

By comparing the Reynolds number of a bubble with the critical value, the presence (or absence) of toroidal wake can then be predicted. This is shown in Figure 10b, where the dimensionless length of the wake, $L_{W} / D$, as a function of $\operatorname{Re}_{-} \operatorname{Re}_{C}$, with $\operatorname{Re}_{C}$ being the critical Reynolds number calculated based on the power correlation obtained in Figure 10a, has been presented. At $R e-R_{C} \leq 0.0, L_{W} / D$ values of 0.0 were obtained, indicating that there is no toroidal wake being formed behind the bubbles in this range. On the other hand, at $0.0<\operatorname{Re}^{-R_{e}} \leq 37.6, L_{W} / D$ can be seen to increase in a linear fashion with 
$R e-R_{C}$. This is in agreement with the observations of Bhaga and Weber (1981), who noted the apparent dependency of the dimensionless length of the wakes on the Reynolds number of the bubble. At higher values of $\operatorname{Re}_{-} \operatorname{Re}_{C}(\geq 79.1)$, the linear dependency of $L_{W} / D$ ceases to exist, and the dimensionless length of the wake appears to decrease with decreasing Reynolds number. This is due to the formation of open and unsteady toroidal wakes behind the bubbles. Further discussion on the extension of open toroidal wakes will be presented in this section.

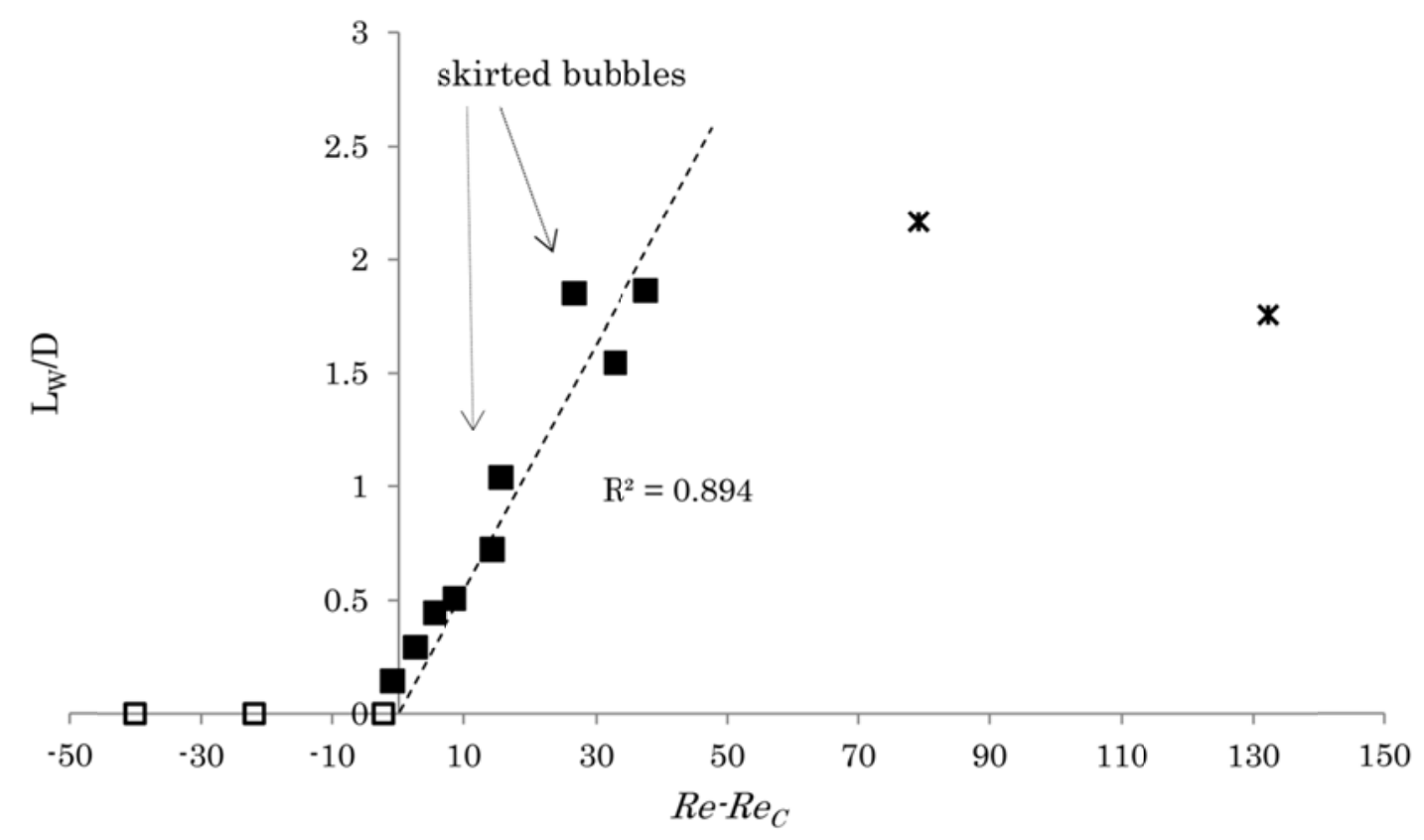

Figure 10b. The length of the wake underneath a rising bubble normalised against the diameter of the bubble. For cases where $\operatorname{Re}-\operatorname{Re}_{C} \leq 37.6$, values at steady state were presented, whereas at

$\operatorname{Re}-\operatorname{Re}_{C}>37.6$, the lengths of the wakes at $0.8 \mathrm{~s}$ were presented. The dotted line indicates a linear fit in the range of $0<\operatorname{Re}^{-R_{e}} \leq 37.6$.

Figure $10 \mathrm{~b}$ also shows that despite the significant differences in the velocity distribution within the primary wakes of skirted bubbles in comparison to those of non-skirted bubbles, the lengths of the wake are still predictable through the Reynolds number of the bubble and fall within the range of the dimensionless wake lengths of non-skirted bubbles.

The extension of an open toroidal (laminar) wake can be inspected through the variations of the normalised axial velocities in Figures 11a and b, respectively, where the growth of the primary and secondary wakes for two different cases, oblate ellipsoidal bubble $[R e=42.2, E o=115, M o=0.1]$ and spherical cap bubble $[R e=94.0, E o=115$, $M o=0.005]$, has been presented. Rather than reaching a steady-state configuration, the primary wakes in the latter case appear to steadily increase in length over time. This is caused by the kinetic energy induced within the wake itself, which is significantly higher in the latter case (Figure 11b). This is a result of the complete separation of the wake from the internal flow of the bubble (see Figure 12); the rotational flow field inside the vortex induces a strong centrifugal force that results in the formation of a region of 
minimum pressure at the vortex centre. The resulting kinetic energy thus needs to be dissipated over a larger wake area, resulting in its expansion over time.

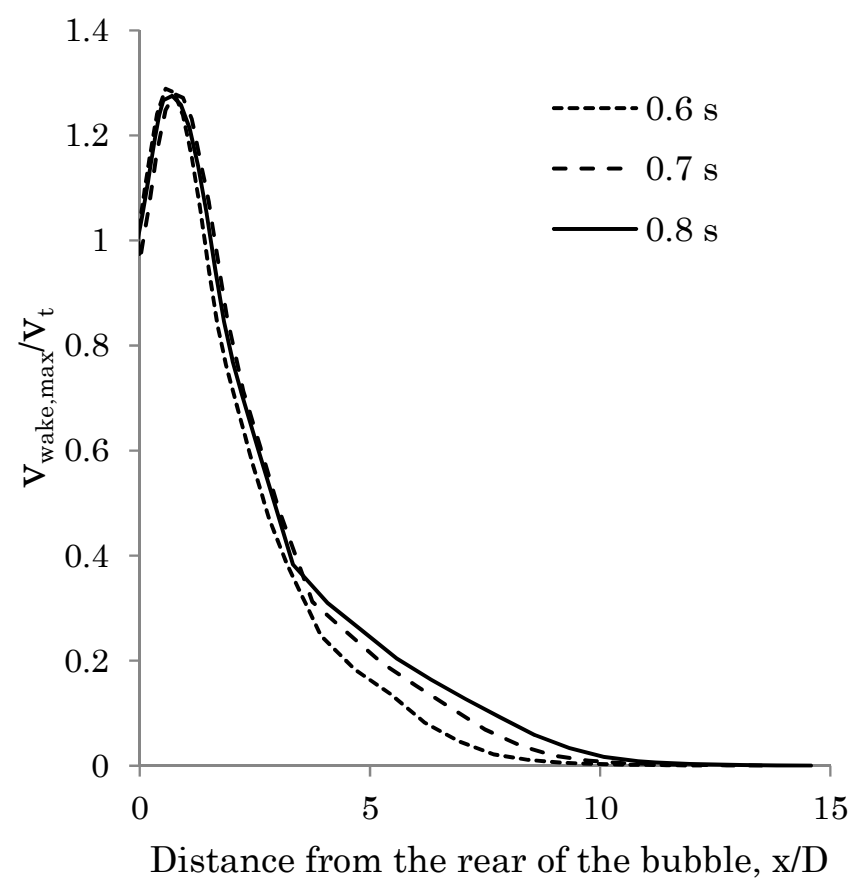

Figure 11a. Development of vertical velocities in the liquid phase along the vertical axis of symmetry of the bubble $[R e=42.2, E o=115, M o=0.1]$.

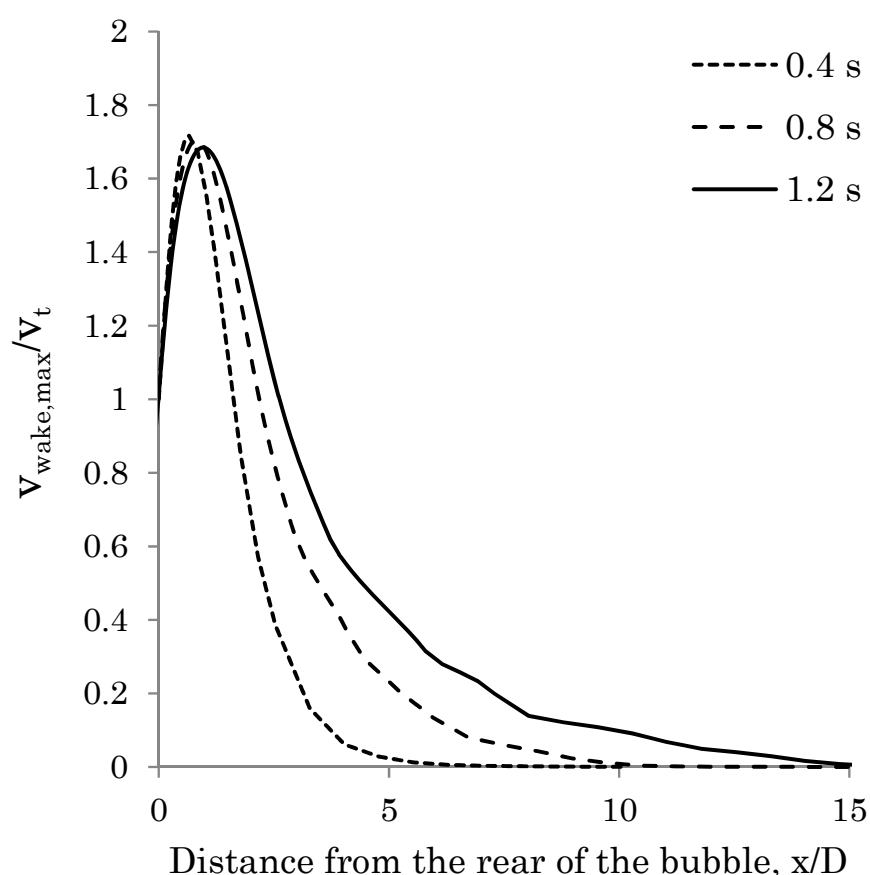

Figure 11b. Development of vertical velocities in the liquid phase along the vertical axis of symmetry of the bubble $[R e=94.0, E o=115, M o=0.005]$. 
The streamlines presented in Figure 12 show that, unlike in previous cases where $R e<94.0$, the flow fields surrounding the bubble is non-axisymmetric, indicating the onset of wake and bubble path instabilities. Even greater instabilities are found to occur in the case of a bubble rising at $R e=151$ and $E o=115$ (see Figure 1n), the velocity field and streamlines of which have been presented in Figure 13. In this case, the instabilities in the bubble wake are compounded by the formation of an additional vortex in the near wake region.

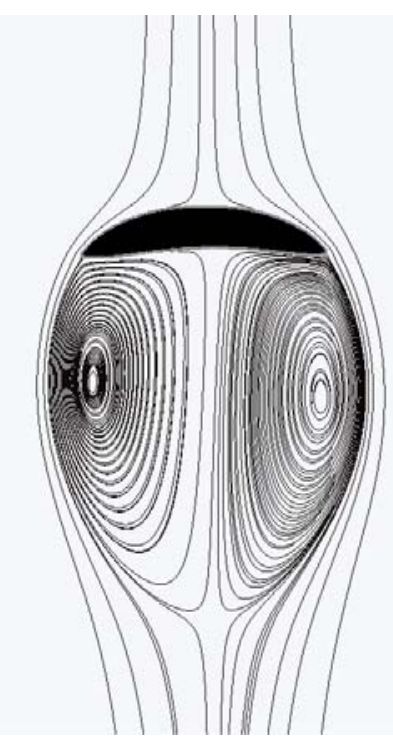

Figure 12. Streamline of the velocity flow field $[E o=641, M o=0.005, R e=94], 1.2$ seconds after the release of the bubble.

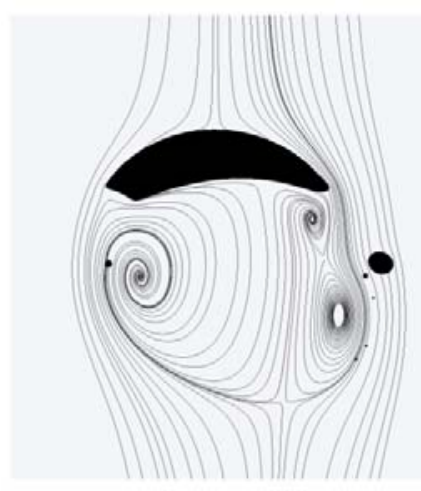

$0.4 \mathrm{~s}$

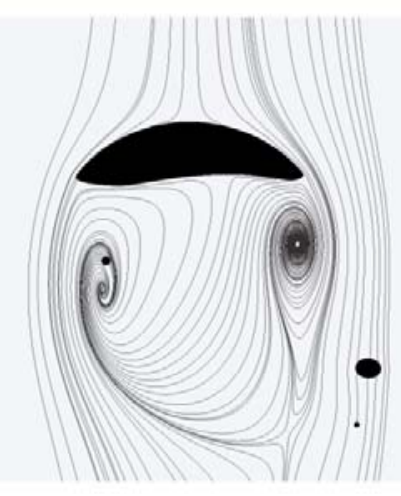

$0.5 \mathrm{~s}$

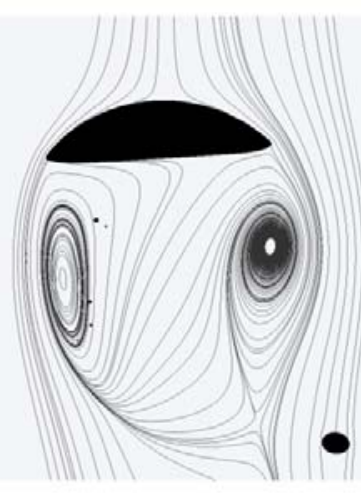

$0.6 \mathrm{~s}$

Figure 13. Streamline of the velocity flow field $[E o=115, M o=0.001, R e=151], 0.4-0.6 \mathrm{~s}$ after the release of the bubble.

The apparent independence of $L_{W} / D$ from $R e-R e_{C}$ in the case of open wake formations, as shown in Figure 10b, is in agreement with the findings of Fan and Tsuchiya (1990), who found that the average size of open unsteady wakes is more constant at high values of Reynolds number. 
The effects of the flow separation and toroidal recirculation at the wake on the path stability of a rising bubble have been studied previously by Cano-Lozano et al. (2013). It was shown that, at high values of $E o$ numbers $(E O \geq 1.5)$, the presence of a standing eddy tends to induce instabilities in the rising path of a bubble, whereas at lower values of $E o(E O<1.5)$, path instabilities in rising bubbles are driven largely by the development of azimuthal vorticity in the boundary layer. In the current case, the path instability of bubbles rising at $E O=115$ has been quantified through the maximum deviation of the radial position of the bubble from its original position in the first $1 \mathrm{~s}$ of bubble rising. This has been presented in Figure 14, where the maximum deviation of the bubble radial position, normalised against the equivalent radius of the bubbles, has been presented against their $R e$ value. In this graph, it is evident that path deviation starts to occur at $R e>13.2$, corresponding to Galileo number of 22.9. The onset of the path instability appears to occur at a lower value of Galileo ( $\mathrm{Ga}$ ) number in comparison to the critical Ga value observed by Cano-Lozano, which at $E O>6$ appears to become constant at $\sim 70$. This could be caused by the significant fore-and-aft asymmetry of dimpled and spherical cap bubbles, resulting from the reduced relative contribution of surface tension in the current case $(E O=115)$.

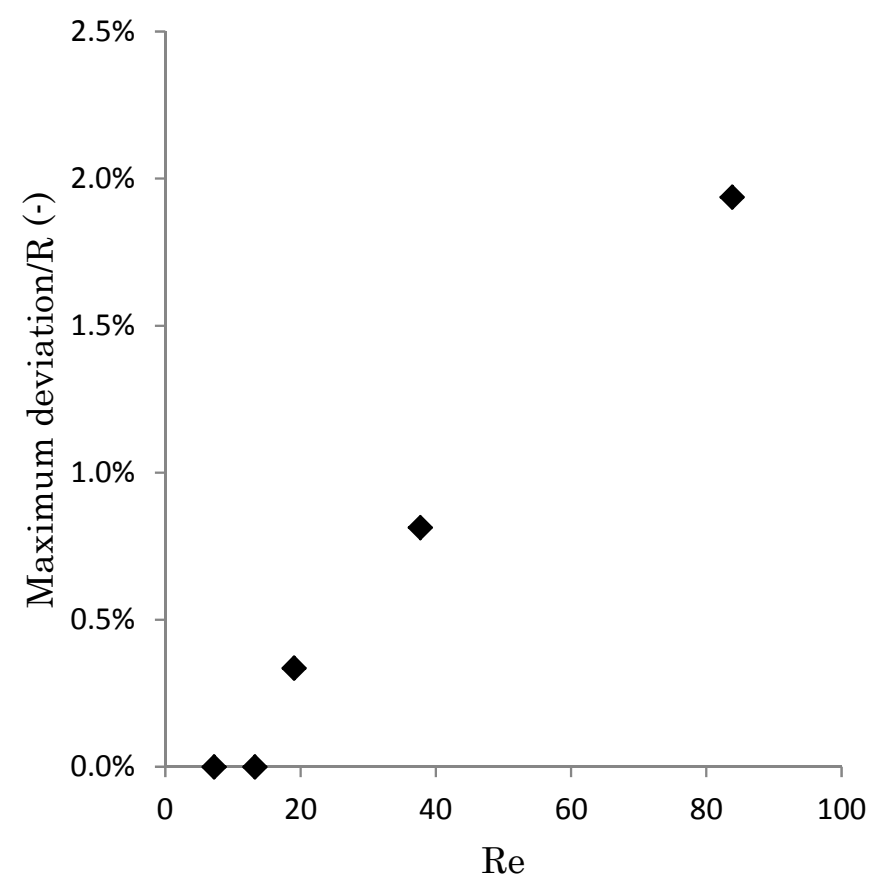

Figure 14. Onset of instability in the rising path of a bubble at $E o=115$.

\subsection{Drag correlation}

As can be seen from Figure 2a, Bhaga \& Weber (1981) tended to over-estimate the terminal velocities of the bubbles. This could be caused by the fluctuations in the bubble rise velocity, which tend to occur in cases where flow separation develops in the wake region. As the experimental determination of the bubble velocity was conducted through the calculation of the distance travelled by a particular bubble in two adjacent frames 
and dividing the distance with the time elapsed, such fluctuations in bubble velocity may have gone unnoticed. As such, in the current study, updated values of the terminal velocity, and the ensuing drag coefficient, have been presented in Figure 15. The drag coefficient for each bubble is calculated as follows:

$$
C_{D}=\frac{F_{D}}{0.5 \rho_{l}\left(\frac{\pi}{4} D^{2}\right) V_{t}^{2}}
$$

The values of the drag coefficient are compared with the correlation proposed by Dijkhuizen et al. (2010), using the corrected values of equivalent bubble diameter,

$$
D_{e q}=\sqrt[3]{\frac{V o l_{\text {leading bubble }}}{\pi / 6}},
$$

for cases where satellite bubbles are formed due to film breakage. Excellent agreement between the simulation results and the correlation of Dijkhuizen et al. (2010) is obtained, with an error margin of $\pm 7 \%$ in the prediction for the bubble terminal velocity. In comparison, the results presented by Bhaga and Weber (1981) generally show lower values of drag coefficients in comparison to the VOF results, particularly at high Reynolds numbers.

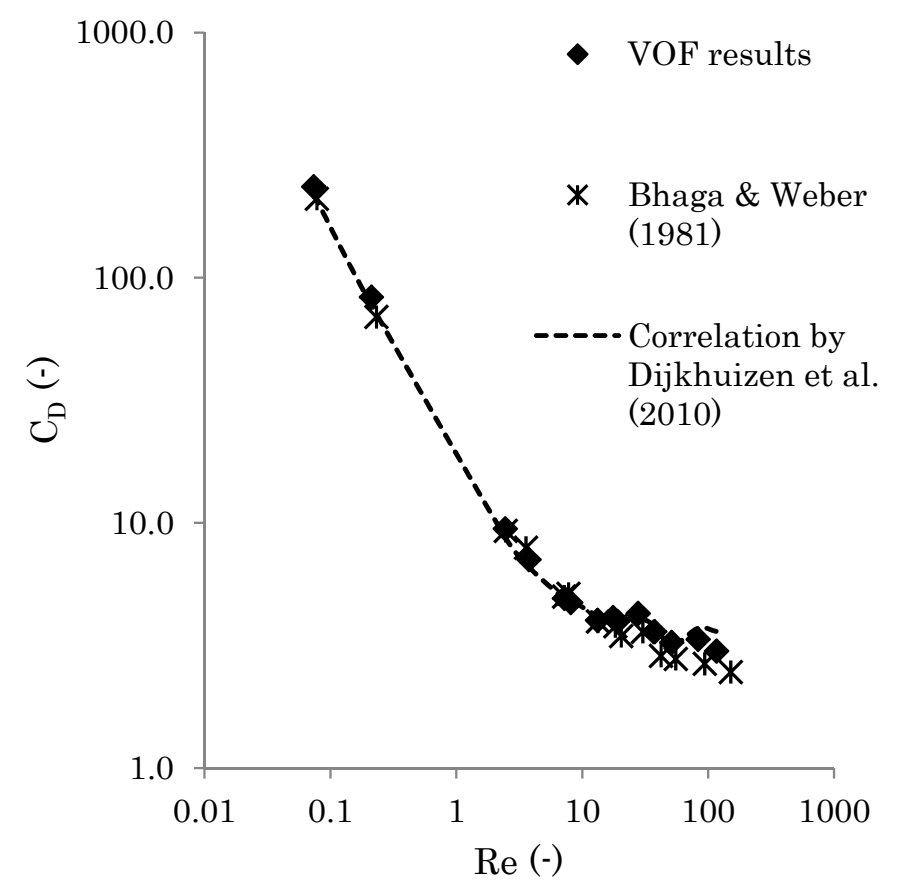

Figure 15. Drag coefficient of the bubbles as a function of Reynolds number. 


\section{Conclusions}

A numerical study on the rise of a bubble in a stagnant liquid has been conducted, encompassing a range of $8.7 \leq E o \leq 641$ and $R e \leq 151$. The bubble is initially spherical and stationary, and, depending on the surface tension and viscous contribution and inertia could deform into ellipsoidal, dimpled, skirted, and spherical cap formations. The requirement for spatial resolution in the simulations is examined, particularly in cases involving high $R e(\approx 90-151)$ and $E o(>115)$ values. In these cases, the formation of thin sections of gas filaments is often encountered, which in turn need to be adequately resolved in computations. It is observed that while the current $3-\mathrm{D}$ simulations still cannot comprehensively reproduce the formation of skirt sections behind a deforming bubble, representative flow fields around the bubbles can still be obtained, such that the rise behaviour of the bubbles reflect the experimental observations of Bhaga and Weber (1981). As a result of this limitation, the formation of satellite bubbles behind a leading bubble rising at high Reynolds number also could not be predicted with good accuracy with the current methodology. At high values of Reynolds numbers, thin gas filaments can also be formed at the top of the bubble, as a result of liquid recirculation upon the separation of the boundary layer at the bottom rim of the bubble. Inadequate resolution of the thin gas filament in this case can result in the misrepresentation of the rupture and reformation of the thin film, which determine the final shape of the bubble; either spherical cap, if the top section of the bubble recovers, or toroidal. In the current study, the formation of a spherical cap bubble with open, unsteady wake at $R e \approx 151$ and $E o \approx 115$, have been presented, in agreement with the experimental observation of Bhaga and Weber (1981). The limitations and requirements for spatial resolution for the study of a bubble rising in a viscous liquid have therefore been identified.

The results of the simulations indicate that the aspect ratio of spherical, ellipsoidal and dimpled bubbles can be predicted through the Morton and Weber numbers of the bubbles. On the other hand, spherical cap bubbles demonstrate a greater dependency of its aspect ratio on the Reynolds number of the bubble. In the case of skirted bubbles, the prediction of the aspect ratio is complicated by the skirt formation itself, and its length is found to be highly dependent on the Capillary number.

The development of toroidal wakes behind the bubble is found to depend largely on the Eo number of the bubble; a correlation is therefore proposed, such that the critical value of Reynolds number, $R e_{C}$, can be predicted as a function of Eo. Bubbles rising at $R e$ values above the corresponding value of $R_{C}$ is found to inhibit toroidal wake formation, and vice versa. Furthermore, a dependency is found on the expansion of the toroidal wake as a function of $R e-R_{C}$. Finally, the formation and development of open toroidal wakes in the case of bubbles rising at $R e>55.3$ are discussed, and analysis on the influence of the wake formation on the onset of path stability of the bubble was presented.

\section{Acknowledgements}

This work was funded through the ARC Discovery Projects Scheme DP140104510. This work was supported by resources provided by The Pawsey Supercomputing Centre with 
funding from the Australian Government and the Government of Western Australia. The authors would also like to acknowledge the remarkable contribution of the Gerris Flow Solver community (http://sourceforge.net/projects/gfs/).

\section{References}

Bhaga, D., Weber, M. E. (1981). Bubbles in viscous liquids: shapes, wakes, and velocities. Journal of Fluid Mechanics 105, 61-85.

Bonometti, T., Magnaudet, J. (2006). Transition from spherical cap to toroidal bubbles. Physics of Fluids 18 (5), 1-12.

Bonometti, T., Magnaudet, J. (2007). An interface-capturing method for incompressible two-phase flows. Validation and application to bubble dynamics. International Journal of Multiphase Flow 33, 109-133.

Cano-Lozano, J. C., Bohorquez, P., Martínez-Bazán, C. (2013). Wake instability of a fixed axisymmetric bubble of realistic shape. International Journal of Multiphase Flow $51,11-21$.

Chen, L., Garimella, S. V., Reizes, J. A., Leonardi, E. (1999). The development of a bubble rising in a viscous liquid. Journal of Fluid Mechanics, 387, 61-96.

Clift, R., Grace, J. R., Weber, M. E. (1978). Bubbles, Drops, and Particles. New York: Academic Press.

Dijkhuizen, W., Roghair, I., Van Sint Annaland, M., Kuipers, J. A. M. (2010). DNS of gas bubbles behaviour using an improved 3D front tracking model-Drag force on isolated bubbles and comparison with experiments. Chemical Engineering Science 65, 14151426 .

Ellingsen, K., Risso, F. (2001). On the rise of an ellipsoidal bubble in water: oscillatory paths and liquid-induced velocity. Journal of Fluid Mechanics 440, 235-368.

Fan, L-S, Tsuchiya, K. (1990). Bubble Wake Dynamics in Liquids and Liquid-Solid Suspensions. Stoneham, MA: Butterworth-Heinemann.

Hua, J., Lou, J. (2007). Numerical simulation of bubble rising in viscous liquid. Journal of Computational Physics 222, 769-795.

Kalra, T. R., Uhlherr, P. H. T. (1971). Properties of bluff-body wakes. $4^{\text {th }}$ Australian Conference on Hydraulics and Fluid Mechanics, Melbourne.

Kulkarni, A. A., Joshi, J. B. (2005). Bubble formation and bubble rise velocity in gas-liquid systems: a review. Industrial \& Engineering Chemistry Research 44, 5873-5931.

Landel, J. R., Cossu, C., Caufield, C. P. (2008). Spherical cap bubbles with a toroidal bubbly wake. Physics of Fluids 20, 122101. 
Lai, H., Yan, Y. Y., Gentle, C. R., (2003). Calculation procedure for conjugate viscous flows about and inside single bubbles. Numerical Heat Transfer, Part B 43, 241-265.

Magnaudet, J., Eames, I. (2000). The motion of high-Reynolds-number bubbles in inhomogeneous flows. Annual Review of Fluid Mechanics 32, 659-708.

Masliyah, J. B., 1972. Steady wakes behind oblate spheroids: flow visualization. Physics of Fluids 15, 1144-1146.

Ohta, M., Imura, T., Yoshida, Y., Sussman, M. (2005). A computational study of the effect of initial bubble conditions on the motion of a gas bubble rising in viscous liquids. International Journal of Multiphase Flow 31, 223-237.

Ohta, M., \& Sussman, M. (2012). The buoyancy-driven motion of a single skirted bubble or drop rising through a viscous liquid. Physics of Fluids (1994-present), 24(11), 112101.

Popinet, S. (2009). An accurate adaptive solver for surface-tension-driven interfacial flows. Journal of Computational Physics 228, 5838-5866.

Ray, B., \& Prosperetti, A. (2014). On skirted drops in an immiscible liquid. Chemical engineering science, 108, 213-222.

Raymond, F., Rosant, J.-M. (2000). A numerical and experimental study of the terminal velocity and shape of bubbles in viscous liquids. Chemical Engineering Science 55, 943955 .

Tripathi, M. K., Sahu, K. C., Govindarajan, R. (2015). Dynamics of an initially spherical bubble rising in quiescent liquid. Nature Communications 6, 6268.

Wairegi, T. (1974). The mechanics of large bubbles and drops moving through extended liquid media (Doctoral dissertation, McGill University).

Walters, J. K., Davidson, J. F. (1963). The initial motion of a gas bubble formed in an inviscid liquid. Part 2. The three-dimensional bubble and the toroidal bubble. Journal of Fluid Mechanics 17, 321-336.

Wu, M., Gharib, M. (2002). Experimental studies on the shape and path of small air bubbles rising in clean water. Physics of Fluids 14, L49.

Zenit, R., Magnaudet, J. (2009). Measurements of the streamwise vorticity in the wake of an oscillating bubble. International Journal of Multiphase Flow 35, 195-203. 